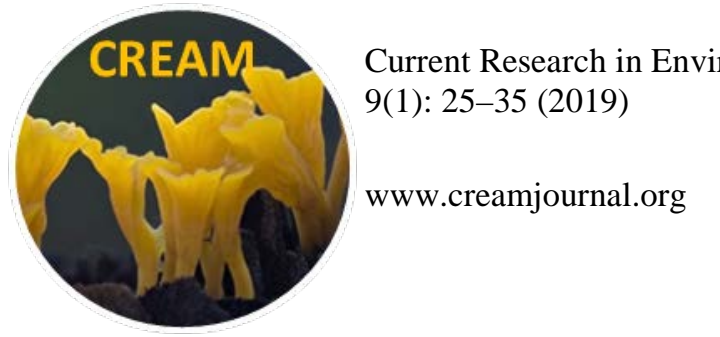

\title{
Fungi associated with forest floor litter in northwest Arkansas
}

\author{
Alanbagi RA ${ }^{1,3_{*}}$, Alshuwaili $\mathrm{FE}^{2,3}$ and Stephenson $\mathrm{SL}^{1}$ \\ ${ }^{1}$ Department of Biological Sciences, University of Arkansas, Fayetteville, Arkansas, 72701, USA. \\ ${ }^{2}$ Department of Plant pathology, University of Arkansas, Fayetteville, Arkansas, 72701, USA. \\ ${ }^{3}$ Department of Horticulture, Green University of Al Qasim, Babylon, Iraq.
}

Alanbagi RA, Alshuwaili FE, Stephenson SL 2019 - Fungi associated with forest floor litter in northwest Arkansas. Current Research in Environmental \& Applied Mycology (Journal of Fungal Biology) 9(1), 25-35, Doi 10.5943/cream/9/1/3

\begin{abstract}
A diverse assemblage of fungi is associated with the litter layer found on the forest floor. Many of these are litter-decomposing fungi, which play a major role as functional decomposers, carbon sequesterers and nutrient immobilizers while also serving as both soil stabilizers and biological remediators. Each year, temperate deciduous forests such as those of northwest Arkansas produce a considerable amount of dead leaves and other types of plant detritus which accumulates on the forest floor. Although it has long been recognized that there is an assemblage of fungi associated with this microhabitat, few studies have been carried out to document the taxa present. The study reported herein represented the first effort of which we are aware to document the fungi associated with forest floor litter in northwest Arkansas. Specimens of fungi collected during the 2016 field season were identified through sequencing of the nuclear ribosomal internal transcribed spacer region (nrDNA-ITS). A total of 127 taxa were recorded, the majority of which were clearly associated with dead leaves. Overall, saprophytes were the dominant ecological group, but the total assemblage also included some taxa with other biological roles (e.g., mycorrhizal fungi).
\end{abstract}

Key words - Forest ecosystems - ITS ribosomal DNA region - litter decomposing fungi taxonomy

\section{Introduction}

The dead leaves and other types of plant detritus which accumulate on the forest floor represent the most valuable renewable resource on the earth. It has been estimated that $90 \%$ of the more than 100 gigatons of terrestrial plant biomass entering the dead organic matter (detritus) pool consists mostly of leaves and other types of plant-derived detritus, including coarse woody debris (Cebrian 1999), and this detritus is the driving force behind the establishment of the soil profile (Berg \& McClaugherty 2003). The dead leaves and other types of non-woody detritus provide microhabitats for the often overlooked litter-decomposing fungi and their consumers in forest ecosystems. These fungi play a major role as functional decomposers, carbon sequesterers and nutrient immobilizers as well serving as both soil stabilizers and biological remediators (Berg \& McClaugherty 2003, Claridge et al. 2009). Moreover, fungi are a major energy input for heterotrophic organisms and are directly involved in nutrient cycling as a result of the decomposition processes in which they are involved (Bödeker et al. 2016, Purahong et al. 2016, Jacobsen et al. 2018). 
Within forest ecosystems, litter-decomposing fungi significantly colonize, enzymatically degrade and efficaciously transform carbon litter materials and immobilize sufficient quantities of detrital nutrients to support their growth and reproduction (Berg \& McClaugherty 2003, Baldrian 2017). Saprophytic fungi are almost exclusively responsible for lignocellulose decomposition, with mycorrhizal fungi also playing some role when they revert to a saprophytic life style (Tedersoo et al. 2014, Bahnmann et al. 2018). These functions connect debris breakdown with overall complex ecosystem processes, and the presence of the fungi allows complex ecological networks involving other organisms, especially invertebrates, to be established (Stephenson 2010, Baldrian 2017, Jacobsen et al. 2018). There is little question that these networks have a considerable impact upon the overall forest ecosystem.

Although litter-decomposing fungi are keystone organisms in the decomposition processes that take place in forest ecosystems, they have rarely been studied to the extent that is true for other groups such as wood-decay fungi and mycorrhizal fungi (Hättenschwiler et al. 2005, Prakash et al. 2015). The study reported herein represented the first effort of which we are aware to document the fungi associated with forest floor litter in the Ozark Mountains of northwest Arkansas. Community composition, microhabitat distribution and putative ecological functions were assessed for the litter-associated fungi in two study areas. These were Pea Ridge National Military Park and Devil's Den State Park. Pea Ridge National Military Park, which covers an area of approximately 1740 ha, is the site of a Civil War battle which occurred in 1862, while Devil's Den State Park consists of about 890 ha and encompasses the largest sandstone crevice area and one of the best preserved Civilian Conservation Corps (CCC) projects in the United States.

\section{Materials \& Methods}

\section{Study areas and collecting}

As noted above, the study areas in the forests of northwest Arkansas were Pea Ridge National Military Park (36 $27^{\prime} 28^{\prime \prime}$ N, 94 $4^{\circ} 01^{\prime} 18^{\prime \prime}$ W, elevation $484 \mathrm{~m}$ ) and Devil's Den State Park (3546’32” N, 94²14'46” W, elevation $454 \mathrm{~m}$ ) in the Ozark Mountains of northwest Arkansas. The forests present in both areas are dominated by an admixture of several species of oak (Quercus alba L., Q. velutina Lam., and Q. marilandica Muenchh.), hickory (Carya spp.), winged elm (Ulmus alata Michx), and red cedar (Juniperus virginiana L.). In addition, red maple (Acer rubrum L.) and sugar maple (Acer saccharum Marshall) occur at Devil's Den, which is the more mesic of the two study areas (Stephenson et al. 2007).

At Pea Ridge, fruiting bodies of fungi were collected from thirty $10 \times 10 \mathrm{~m}$ study plots in the manner described in Lodge et al. (2004). The plots were located along a transect and placed 35-50 $\mathrm{m}$ apart in order to minimize sampling the same genet frequently and also to obtain fruitings from a wide variety of microsites on the forest floor. The corners of the plots were delimited with stake wire flags. At Devil's Den, areas of forest floor litter were examined in an opportunistic manner as described by Cannon \& Sutton (2004), and any fruiting bodies of fungi associated with this microhabitat were collected.

\section{Processing and identification of fruiting bodies}

Fruiting bodies were collected directly from the litter layer on the forest floor in these plots on a series of visits that took place at approximately two or three week intervals during the period of May to September 2016. After recording substrate data, fruiting bodies were photographed in their natural habitats, placed in the compartments of a plastic fishing tackle box and transferred to the laboratory. The substrates upon which fruiting bodies occurred were segregated into three microhabitat categories. These were leafy litter (LL), consisting of dead leaves present on the forest floor; woody litter (WL), consisting of small twigs ( $<2 \mathrm{~cm}$ in diameter), small pieces of woody debris, and tiny fragments of bark (no more than $0.5-2.5 \mathrm{~cm}$ in diameter); and mixed litter (ML) representing instances in which the mycelium associated with a fruiting body proliferated across both leafy and woody substrates or the fruiting bodies were collected from a combination of LL and WL. 
Fruiting bodies were dried on a food dehydrator at a temperature $45{ }^{\circ} \mathrm{C}$ for at least $24 \mathrm{~h}$, placed in small pasteboard boxes and then deposited in the mycological herbarium of the University of Arkansas (UARK) for future study. The morphological characteristics of fruiting bodies were recorded in either the field or laboratory for use in identification, and the latter was accomplished with the use of appropriate keys and various other publications (e.g., Breitenbach \& Kränzlin 1984, 1995, Binion et al. 2008, Elliott \& Stephenson 2018). Later, identifications were confirmed by amplifying the internal transcribed spacer (ITS) region of the fungal ribosomal DNA (rDNA).

In another component of the project described herein, samples of forest floor litter consisting mostly of fallen leaves were collected from the four corners and the center of each investigated plot in May 2016, pooled together and placed in plastic incubation chambers $(31.5 \mathrm{~cm}$ long $\times 17.5 \mathrm{~cm}$ wide $\times 10.5 \mathrm{~cm}$ tall). These incubation chambers were maintained with adequate moisture via spraying the surface of the sample material with water in order to induce the growth and development of fungi. At regular intervals, the incubation chambers were checked for the presence of fruiting bodies, and these were collected, conserved and processed in the same manner as fruiting bodies collected in the field.

\section{DNA extraction and amplification}

Genomic DNA was extracted from small fragments (usually taken from the pileus or stipe) of selected fruiting bodies and processed with the use of the Wizard Genomic DNA purification Kit (Promega, Madison, Wisconsin), following the manufacturer's protocol. The extracted DNA was amplified and sequenced using the fungal specific primer pairs ITS1F-ITS4 or ITS4B (Gardes \& Bruns 1993) along with the universal primers ITS1 and ITS4 (White et al. 1990). Polymerase chain reactions were carried out with $2 \mathrm{X}$ Go Taq Green master mix and amplification was carried out with a BioRed T100 ${ }^{\mathrm{TM}}$ thermal cycler. The PCR protocol of specific primer pairs involved a hot start at $95{ }^{\circ} \mathrm{C}$ for $5 \mathrm{~min}$, followed by 37 cycles of $30 \mathrm{~s}$ denaturation at $95{ }^{\circ} \mathrm{C}, 30 \mathrm{~s}$ annealing at $56{ }^{\circ} \mathrm{C}, 1 \mathrm{~min}$ amplification at $72{ }^{\circ} \mathrm{C}$, and a final 5 min elongations at $72{ }^{\circ} \mathrm{C}$. Similar preheated and final elongated conditions were applied for the primers ITS1-ITS4 in the thermal cycler program with performing 35 cycles of $45 \mathrm{~s}$ denaturation at $96{ }^{\circ} \mathrm{C}, 45 \mathrm{~s}$ annealing at $52{ }^{\circ} \mathrm{C}, 1.30 \mathrm{~min}$ amplification at $72{ }^{\circ} \mathrm{C}$.

The size and purity of PCR products were assessed via electrophoresis and resolved on $1 \%$ agarose gel in 0.5X TAE buffer, stained by SYBR safe, and sized using the DNA Ladder (QuickLoad ${ }^{\circledR}$, New England BioLabs, Ipswich, Massachusetts). Afterwards, amplified bands were visualized and photographed with an ultraviolet transilluminator using a Gel-Doc image analysis system.

\section{Sanger sequencing and taxonomic determinations}

The successfully amplified products were sent for direct Sanger sequencings by GENEWIZ (Boston, Massachusetts) after enzymatical clean-up of the PCR sequencing templates. The resulting sequences were edited and aligned with the Geneious program version 9.1.8 (Biomatters Ltd., Newark, New Jersey) to obtain a consensus sequence after manually correcting the alignment errors. For a verified identity, each sequence fragment was subjected to an individual Basic Local Alignment Search Tool (BLAST) available at the National Center for Biotechnology Information (NCBI) GenBank (www.ncbi.nlm.nih.gov/genbank/).

Determining the region of similarity and then comparing and calculating the statistical significances between fungal nucleotide sequences was completed using query coverage of $\geqslant 80 \%$ and $\geqslant 97-100 \%$ sequence similarity based on consideration of the results from the GenBank BLAST search. The taxonomic level and current taxonomic names for the taxa of fungi listed herein follow those given on Index Fungorum (www.indexfungorum.org). Each identified taxon was putatively assigned to a particular ecological functional group based on information available in the relevant literature (e.g., Rinaldi et al. 2008, Tedersoo et al. 2014). 


\section{Results}

\section{Taxonomic diversity}

A total of 127 taxa of litter-associated fungi were recorded from the 2585 fruiting bodies collected and/or recorded from the two study areas and the incubation chambers (Table 1). As noted, these taxa were taxonomically identified, first from morphological characters and then this identification was confirmed genetically from ITS sequence data. These 127 taxa represented 63 genera, at least 37 families and 15 orders. The majority of the identified taxa belong to the families Marasmiaceae (15 taxa), Inocybaceae (14 taxa), Omphalotaceae (14 taxa), Inocybaceae (14 taxa), Entolomataceae (10 taxa) and Mycenaceae (10 taxa) in the phylum Basidiomycota. However, several families such as the Lachnaceae, Rutstroemiaceae, Sarcoscyphaceae, Orbiliaceae and Xylariaceae in the phylum Ascomycota also were identified (Table 1, Fig. 1). The members of a particular family tend to be associated with a different type of litter material (e.g., small twigs versus dead leaves), and those examples collected from dead leaves and mixed litter were the most common (Table 1). As can be noted in Table 1, there were an appreciable number of specimens which yielded sequences that could not be matched beyond genus with anything in GenBank. It seems likely that the majority of these are either relatively rare taxa yet to be sequenced or taxa new to science.

\section{Functional diversity}

Saprophytes made up the single most dominant ecological functional group on litter materials in the temperate deciduous forests considered in the present study, although an ecological function could not be assigned to a number of taxa (Fig. 2). Most of the saprophytic taxa belong to the Omphalotaceae, Marasmiaceae, Agaricaceae and Entolomataceae, all of which were collected predominantly from leafy and woody litter materials. The majority of these taxa produce relatively small (albeit visible to the naked eye and thus possible to collect in the field) fruiting bodies (Fig. 3).

\section{Discussion}

The research described herein produced a comprehensive body of data on what is an understudied assemblage of fungi-those species associated with forest floor litter. The total of 127 taxa, all of which produce macroscopic fruiting bodies, clearly exhibited a high level of taxonomic diversity, since they were assigned to 63 genera, at least 37 families and 15 orders (Table 1). This was not surprising, since a high level of biodiversity has been reported in a number of other studies (e.g., Kubartová et al. 2009, Bahnmann et al. 2018). Forest floor litter is a heterogenous microhabitat consisting of a mixture of several different types of detritus (e.g., dead leaves, small twigs, and tiny pieces of bark) which provide substrates with various chemical and physical properties that presumably represent different niches for fungi to exploit (Berg \& McClaugherty 2003, Elliott \& Stephenson 2018). Some fungi are likely to display a preference for detritus from particular kinds of trees, so the overall biodiversity of the forest almost surely represents a factor accounting for the number of taxa present (Hattenschwiler et al. 2005).

Fungi that decompose dead leaves were the group characterized by the greatest abundance and richness (Table 1) in the litter microhabitat. Newly fallen leaves provide relatively nutrient-rich and thus favorable substrates for fungi, and once established, their mycelia grow and develop. The presence of the mycelia provides a setting for ecological interactions with bacteria and invertebrates (Boddy et al. 2008, Hardoim et al. 2015, Prakash et al. 2015). To what extent these interactions influence the structure and composition of the assemblages of fungi associated with litter is not yet known. Presumably, the stage of litter decomposition also represents a factor in determining just what taxa of fungi are present on a particular substrate. It has been suggested that as fungi colonize and enzymatically degrade and assimilate the preferred or available biopolymers, the differences in the metabolic abilities which exist from taxon to taxon contribute to the overall fungal biodiversity (Purahong et al. 2016). All of these factors underscore the complexity of the 
fungus-substrate relationships associated with forest floor litter, including the manner in which the fungal mycelium, once established, is able to colonize new substrates.

Table 1 Fungi identified associated with the litter microhabitat in the forests of northwest Arkansas as identified from ITS sequences. Note: $\mathrm{MH}=$ microhabitat $(\mathrm{LL}=$ leafy litter, $\mathrm{WL}=$ woody litter and $\mathrm{ML}=$ mixed litter), ID = percent $(\%)$ sequence identity, $\mathrm{QC}=$ percent $(\%)$ query coverage of the sequences in GenBank.

\begin{tabular}{|c|c|c|c|c|c|}
\hline Family & Taxon & MH & $\begin{array}{l}\text { Accession } \\
\text { number }\end{array}$ & ID & QC \\
\hline & Phylum Ascomycota & & & & \\
\hline Hyaloscyphaceae & Incrucipulum ciliare (Schrad.) Baral* & LL & KT876985 & 99 & 100 \\
\hline Hypocreaceae & $\begin{array}{l}\text { Trichoderma hypoxylon Jing Z. Sun, Xing Z. } \\
\text { Liu \& K.D. Hyde* }\end{array}$ & LL & KU974002 & 98 & 88 \\
\hline Lachnaceae & Lachnum brevipilosum Baral & WL & AB267643 & 99 & 91 \\
\hline Lachnaceae & Lachnum virgineum (Batsch) P. Karst. & WL & AB481268 & 99 & 99 \\
\hline Orbiliaceae & Orbilia luteorubella (Nyl.) P. Karst. & WL & U72607 & 99 & 91 \\
\hline Parmeliaceae & Punctelia hypoleucites (Nyl.) Krog & WL & HQ650685 & 99 & 93 \\
\hline Pezizaceae & Peziza succosa Berk. & WL & MG663289 & 99 & 100 \\
\hline Pyronemataceae & Pyronemataceae (unknown species) & ML & KJ209693 & 82 & 100 \\
\hline Pyronemataceae & Humaria sp. 1 & ML & MG019762 & 99 & 93 \\
\hline Rutstroemiaceae & Rutstroemia sp. 1 & LL & AB926083 & 91 & 99 \\
\hline Rutstroemiaceae & Rutstroemia sp. 2 & LL & LT158447 & 93 & 98 \\
\hline Sarcosomataceae & Galiella rufa (Schwein.) Nannf. \& Korf & WL & AF485073 & 99 & 93 \\
\hline Sclerotiniaceae & Moellerodiscus sp. 1 & LL & AB926070 & 95 & 98 \\
\hline Sarcoscyphaceae & Sarcoscypha occidentalis Schwein.) Sacc. & WL & MF992165 & 99 & 99 \\
\hline Xylariaceae & Xylaria cornu-damae (Schwein.) Berk. & WL & MH859400 & 99 & 94 \\
\hline Xylariaceae & Xylaria hypoxylon (L.) Grev. & ML & AY327477 & 99 & 96 \\
\hline Xylariaceae & Xylaria sp. 1 & ML & JQ761773 & 99 & 94 \\
\hline \multirow[t]{2}{*}{ Xylariaceae } & Xylaria sp. 2 & LL & GU300082 & 99 & 95 \\
\hline & Phylum Basidiomycota & & & & \\
\hline Agaricaceae & Crucibulum laeve (Huds.) Kambly & WL & DQ071701 & 98 & 99 \\
\hline Agaricaceae & Cyathus sp. 1 & ML & NR_119589 & 96 & 94 \\
\hline Agaricaceae & Cystolepiota seminuda (Lasch) Bon & ML & AY176350 & 97 & 93 \\
\hline Agaricaceae & Lepiota sp. 1 & LL & HQ647294 & 90 & 95 \\
\hline Agaricaceae & Lepiota sp. 2 & LL & MH211803 & 100 & 84 \\
\hline Agaricaceae & Leucoagaricus sp. 1 & LL & MG050099 & 99 & 80 \\
\hline Agaricaceae & Lycoperdon perlatum Pers. & ML & КР340193 & 99 & 100 \\
\hline Amanitaceae & Amanita bisporigera G.F. Atk. & LL & EU819411 & 99 & 100 \\
\hline Amanitaceae & Amanita sp. 1 & LL & KP711841 & 97 & 99 \\
\hline Bolbitiaceae & Conocybe pubescens (Gillet) Kühner & ML & MH855752 & 99 & 98 \\
\hline Bolbitiaceae & Conocybe sp. 1 & WL & JF907826 & 95 & 100 \\
\hline Bolbitiaceae & Conocybe nigrescens Hauskn. \& Gubitz & WL & JX968234 & 98 & 93 \\
\hline Bolbitiaceae & Galerella nigeriensis Tkalčec, Mešić \& Čerkez & ML & JX968251 & 98 & 90 \\
\hline Cortinariaceae & Cortinarius hinnuleoarmillatus Reumaux & LL & DQ499460 & 97 & 100 \\
\hline Entolomataceae & $\begin{array}{l}\text { Clitocella popinalis (Fr.) Kluting, T.J. Baroni } \\
\text { \& Bergemann }\end{array}$ & LL & FJ770397 & 98 & 97 \\
\hline Entolomataceae & Clitopilus sp. 1 & WL & KC176282 & 94 & 100 \\
\hline Entolomataceae & Clitopilus sp. 2 & WL & NR_137867 & 96 & 99 \\
\hline Entolomataceae & Clitopilus sp. 3 & WL & NR_137867 & 94 & 100 \\
\hline Entolomataceae & Clitopilus sp. 4 & ML & KU862859 & 88 & 92 \\
\hline Entolomataceae & Entoloma rhodocylix (Lasch) M.M. Moser & LL & KJ001414 & 99 & 86 \\
\hline Entolomataceae & Entoloma sp. 1 & LL & KX387621 & 90 & 93 \\
\hline Entolomataceae & Entoloma sp. 2 & WL & JF908003 & 89 & 98 \\
\hline Entolomataceae & Entoloma sp. 3 & WL & JF908007 & 92 & 100 \\
\hline
\end{tabular}


Table 1 Continued.

\begin{tabular}{|c|c|c|c|c|c|}
\hline Family & Taxon & MH & $\begin{array}{l}\text { Accession } \\
\text { number }\end{array}$ & ID & QC \\
\hline Entolomataceae & Entoloma sp. 4* & LL & KX387621 & 96 & 95 \\
\hline Hydnangiaceae & Laccaria bicolor (Maire) P.D. Orton & LL & KM067816 & 99 & 100 \\
\hline Hydnangiaceae & Laccaria laccata (Scop.) Cooke & LL & KY744187 & 99 & 100 \\
\hline Hymenochaetaceae & Coltricia confluens P.-J. Keizer & WL & NR_137867 & 99 & 97 \\
\hline Incertae sedis & $\begin{array}{l}\text { Resinicium pinicola (J. Erikss.) J. Eriksss \& } \\
\text { Hjortstam* }\end{array}$ & WL & KJ668463 & 98 & 100 \\
\hline Inocybaceae & Crepidotus sp. 1 & WL & MF461325 & 87 & 63 \\
\hline Inocybaceae & Crepidotus sp. 2 & LL & KF830099 & 86 & 100 \\
\hline Inocybaceae & Inocybe grammata Quél. & LL & GQ166896 & 99 & 99 \\
\hline Inocybaceae & Inocybe radiata Peck & LL & GU819490 & 99 & 100 \\
\hline Inocybaceae & Inocybe subfulva Peck & ML & KP641623 & 99 & 99 \\
\hline Inocybaceae & Inocybe subradiata Murrill & ML & MF992157 & 99 & 99 \\
\hline Inocybaceae & Inocybe sp. 1 & ML & KJ432283 & 89 & 100 \\
\hline Inocybaceae & Inocybe sp. 2 & LL & KY990545 & 90 & 97 \\
\hline Inocybaceae & Inocybe sp. 3 & ML & MF992163 & 99 & 95 \\
\hline Inocybaceae & Inocybe sp. 4 & ML & HQ604204 & 90 & 97 \\
\hline Inocybaceae & Inocybe sp. 5 & LL & NR_153163 & 81 & 64 \\
\hline Inocybaceae & Inocybe sp. 6 & LL & JF908124 & 94 & 99 \\
\hline Inocybaceae & Phaeomarasmius sp. & WL & MH856667 & 94 & 98 \\
\hline Inocybaceae & Simocybe sumptuosa (P.D. Orton) Singer & WL & KT715798 & 98 & 99 \\
\hline Lyophyllaceae & Lyophyllum sp. 1 & LL & MF773643 & 99 & 99 \\
\hline Marasmiaceae & Crinipellis nigricaulis Har.Takah * & LL & FJ573197 & 97 & 100 \\
\hline Marasmiaceae & Crinipellis setipes (Peck) Singer * & LL & JF930641 & 99 & 100 \\
\hline Marasmiaceae & Crinipellis zonata (Peck) Sacc. & WL & MH979260 & 99 & 99 \\
\hline Marasmiaceae & $\begin{array}{l}\text { Gerronema subclavatum (Peck) Singer ex } \\
\text { Redhead }\end{array}$ & WL & U66434 & 99 & 99 \\
\hline Marasmiaceae & Marasmius capillaris Morgan* & LL & MH979289 & 99 & 100 \\
\hline Marasmiaceae & $\begin{array}{l}\text { Marasmius conchiformis J.S. Oliveira \& } \\
\text { Capelari }\end{array}$ & LL & KF741996 & 99 & 96 \\
\hline Marasmiaceae & Marasmius pulcherripes Peck & LL & MF161270 & 99 & 82 \\
\hline Marasmiaceae & Marasmius rotula (Scop.) Fr. & WL & DQ182506 & 99 & 98 \\
\hline Marasmiaceae & Marasmius siccus (Schwein.) Fr. & LL & HQ607384 & 99 & 100 \\
\hline Marasmiaceae & Marasmius sp. 1 & LL & KY366495 & 91 & 99 \\
\hline Marasmiaceae & Marasmius sp. 2 & LL & KF774157 & 94 & 100 \\
\hline Marasmiaceae & Marasmius sp. 3* & LL & KP826788 & 87 & 70 \\
\hline Marasmiaceae & Marasmius sp. 4 & WL & KP013041 & 96 & 97 \\
\hline Marasmiaceae & Trogia sp. 1 & LL & MF100962 & 86 & 96 \\
\hline Marasmiaceae & Trogia sp. 2 & ML & AY329595 & 87 & 99 \\
\hline Meruliaceae & Gloeoporus dichrous (Fr.) Bres. & WL & MG748583 & 99 & 100 \\
\hline Meruliaceae & Steccherinum sp. 1 & WL & KP814318 & 99 & 100 \\
\hline Mycenaceae & Нетітусепа sp. 1 & LL & MH856249 & 93 & 93 \\
\hline Mycenaceae & Нетітусепа sp. 2 & LL & MK169368 & 91 & 99 \\
\hline Mycenaceae & Mycena albiceps (Peck) Gilliam* & LL & KY744172 & 99 & 83 \\
\hline Mycenaceae & Mycena citrinomarginata Gillet* & LL & GU234150 & 99 & 100 \\
\hline Mycenaceae & Mycena pearsoniana Dennis & ML & JN182200 & 99 & 91 \\
\hline Mycenaceae & Mycena pura (Pers.) P. Kumm. & ML & MF955190 & 98 & 100 \\
\hline Mycenaceae & Mycena stylobates (Pers.) P. Kumm.* & LL & JF908439 & 99 & 89 \\
\hline Mycenaceae & Mycena sp. 1 & LL & MH136827 & 86 & 95 \\
\hline Mycenaceae & Mycena sp. 2 & LL & JF908384 & 90 & 99 \\
\hline Mycenaceae & Mycena sp. 3 & ML & LT671449 & 87 & 100 \\
\hline Mycenaceae & Mycena sp. 4 & WL & DQ490645 & 86 & 100 \\
\hline Omphalotaceae & $\begin{array}{l}\text { Gymnopus androsaceus (L.) Della Magg. \& } \\
\text { Trassin }\end{array}$ & LL & MH857176 & 97 & 98 \\
\hline Omphalotaceae & $\begin{array}{l}\text { Gymnopus cremeostipitatus Antonín, Ryoo \& } \\
\text { Ka }\end{array}$ & LL & NR_152898 & 98 & 96 \\
\hline
\end{tabular}


Table 1 Continued.

\begin{tabular}{|c|c|c|c|c|c|}
\hline Family & Taxon & MH & $\begin{array}{l}\text { Accession } \\
\text { number }\end{array}$ & ID & QC \\
\hline Omphalotaceae & $\begin{array}{l}\text { Gymnopus dichrous (Berk. \& M.A. Curtis) } \\
\text { Halling }\end{array}$ & LL & KY026696 & 97 & 99 \\
\hline Omphalotaceae & $\begin{array}{l}\text { Gymnopus disjunctus R.H. Petersen \& K.W. } \\
\text { Hughes }\end{array}$ & LL & NR_137865 & 99 & 99 \\
\hline Omphalotaceae & Gymnopus foliiphilus R.H. Petersen & LL & KY026633 & 100 & 100 \\
\hline Omphalotaceae & $\begin{array}{l}\text { Gymnopus junquilleus R.H. Petersen \& J. L. } \\
\text { Mata }\end{array}$ & LL & AY256693 & 99 & 98 \\
\hline Omphalotaceae & $\begin{array}{l}\text { Gymnopus spongiosus (Berk. \& M. A. Curtis) } \\
\text { Halling }\end{array}$ & WL & AF505784 & 99 & 96 \\
\hline Omphalotaceae & Gymnopus subnudus (Ellis ex Peck) Halling & ML & KY777383 & 99 & 99 \\
\hline Omphalotaceae & Gymnopus sp. 1 & LL & KY026619 & 99 & 99 \\
\hline Omphalotaceae & $\begin{array}{l}\text { Marasmiellus rhizomorphigenus Antonín, Ryoo } \\
\text { \& H.D. Shin }\end{array}$ & LL & GU319116 & 99 & 86 \\
\hline Omphalotaceae & Marasmiellus sp. 1 & WL & MF161165 & 98 & 100 \\
\hline Omphalotaceae & $\begin{array}{l}\text { Mycetinis copelandii (Peck) A.W. Wilson \& } \\
\text { Desjardin* }\end{array}$ & LL & KY696751 & 98 & 100 \\
\hline Omphalotaceae & $\begin{array}{l}\text { Mycetinis opacus (Berk. \& M.A. Curtis) A.W. } \\
\text { Wilson \& Desjardin }\end{array}$ & ML & MG663278 & 99 & 100 \\
\hline Omphalotaceae & $\begin{array}{l}\text { Mycetinis scorodonius (Fr.) A.W. Wilson \& } \\
\text { Desjardin }\end{array}$ & WL & JQ272364 & 99 & 100 \\
\hline Physalacriaceae & Strobilurus sp. & LL & HQ604789 & 91 & 100 \\
\hline Pluteaceae & Pluteus leoninus sensu Rea, Cooke & WL & HM562190 & 100 & 97 \\
\hline Polyporaceae & Trametes conchifer (Schwein.) Pilát & WL & JN164939 & 99 & 97 \\
\hline Psathyrellaceae & Coprinellus sp. 1 & LL & MH856811 & 96 & 99 \\
\hline Psathyrellaceae & $\begin{array}{l}\text { Coprinellus xanthothrix (Romagn.) Vilgalys, } \\
\text { Hopple \& Jacq. Johnson }\end{array}$ & WL & KJ028784 & 99 & 96 \\
\hline Psathyrellaceae & $\begin{array}{l}\text { Coprinus sclerocystidiosus (M. Lange \& A.H. } \\
\text { Sm.) Vilgalys, Hopple \& Jacq. Johnson }\end{array}$ & WL & KC992942 & 99 & 100 \\
\hline Psathyrellaceae & Psathyrella candolleana (Fr.) Maire & WL & HQ436117 & 98 & 100 \\
\hline Russulaceae & Lactarius camphoratus (Bull.) Fr. & ML & EU819480 & 97 & 100 \\
\hline Russulaceae & Lactarius pterosporus Romagn. & LL & KF432963 & 98 & 99 \\
\hline Russulaceae & Lactarius subserifluus Longyear & WL & EU819486 & 98 & 100 \\
\hline Schizophyllaceae & Schizophyllum commune Fr. & WL & МН307932 & 99 & 100 \\
\hline Sclerodermataceae & Scleroderma areolatum Ehrenb. & WL & EU819438 & 99 & 100 \\
\hline Sebacinaceae & Sebacina candida L.S. Olive & ML & KF061277 & 99 & 99 \\
\hline Sebacinaceae & Tremellodendron schweinitzii (Peck) G.F. Atk. & LL & KY744167 & 97 & 98 \\
\hline Strophariaceae & Deconica sp. 1 & WL & KM270756 & 95 & 99 \\
\hline Thelephoraceae & Thelephora anthocephala (Bull.) Fr. & WL & AF272927 & 98 & 86 \\
\hline Thelephoraceae & Thelephora sp. & WL & KP783471 & 93 & 99 \\
\hline Tremellaceae & $\begin{array}{l}\text { Tremella yokohamensis (Alshahni, Satoh \& } \\
\text { Makimura) Yurkov }\end{array}$ & WL & KP986529 & 97 & 97 \\
\hline Tricholomataceae & Clitocybe subditopoda Peck & ML & KM453734 & 99 & 99 \\
\hline Tricholomataceae & Clitocybe sp. 1 & ML & KJ680984 & 86 & 100 \\
\hline Tricholomataceae & Infundibulicybe gibba (Pers.) Harmaja & LL & MG663274 & 99 & 100 \\
\hline Tricholomataceae & Resupinatus alboniger (Pat.) Singer & WL & КР026234 & 99 & 99 \\
\hline Tubariaceae & Tubaria sp. 1 & ML & MF039263 & 97 & 97 \\
\hline Tubariaceae & Tubaria sp. 2 & LL & MF039263 & 87 & 96 \\
\hline
\end{tabular}

Note: Names in bold represent taxa recorded both in the field and from incubation chambers, whereas “*” indicates taxa recorded only from incubation chambers. 


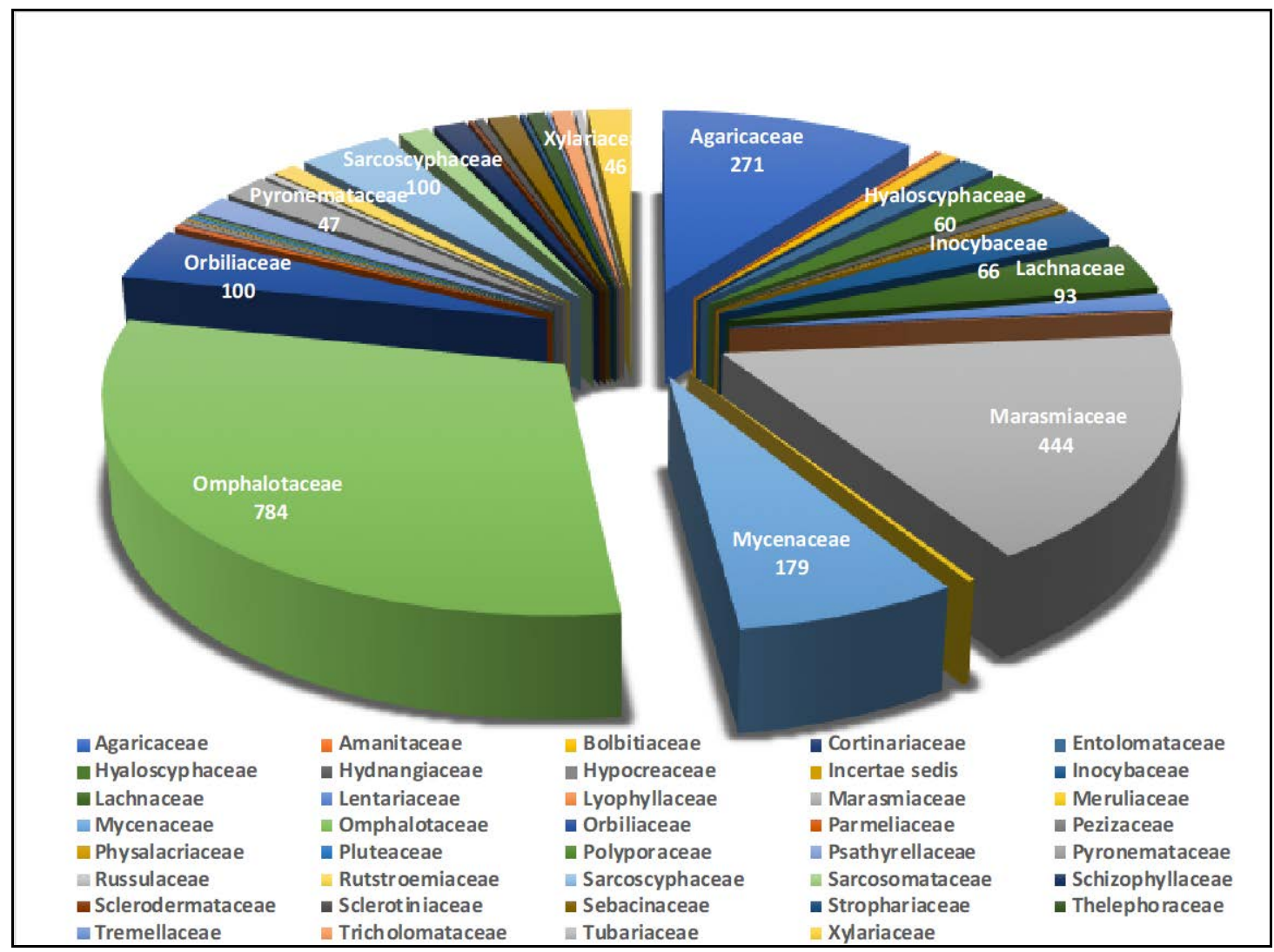

Fig. 1 - Families represented by litter-associated fungi recorded in the present study. The numbers reflect their relative abundance in the litter microhabitat.

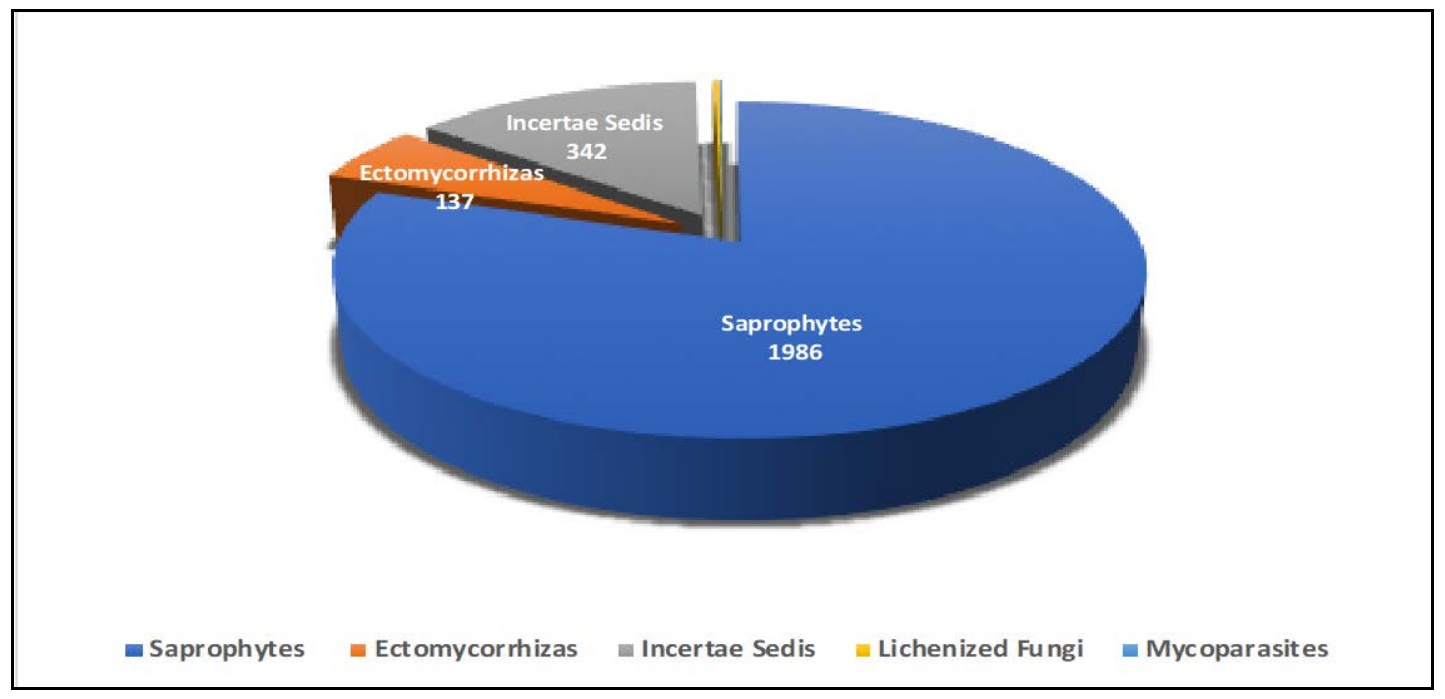

Fig. 2 - Relative abundance of litter-associated fungi in the major ecological functional groups.

In the present study, as already noted, the taxa recorded from the litter microhabitat were assigned to ecological functional groups (Fig. 2). Saprophytes, with a total of 1986 fruiting bodies were clearly the most abundant fungi present. These fungi undoubted possess the various functional characters that allow them to exploit the types of plant detritus that make up litter (Goswami et al. 2017). However, fungi representing other functional groups also were recorded, thus providing evidence of the functional overlap that exists between fungal saprophytes and fungi with different ecological roles. For example, 137 of the fruiting bodies collected were those of ectomycorrhizal fungi, which not only use forest floor litter as a substrate for fruiting, but also have a role as organic 
decomposers to meet some of their energy needs (Rinaldi et al. 2008, Bödeker et al. 2016, Bahnmann et al. 2018).

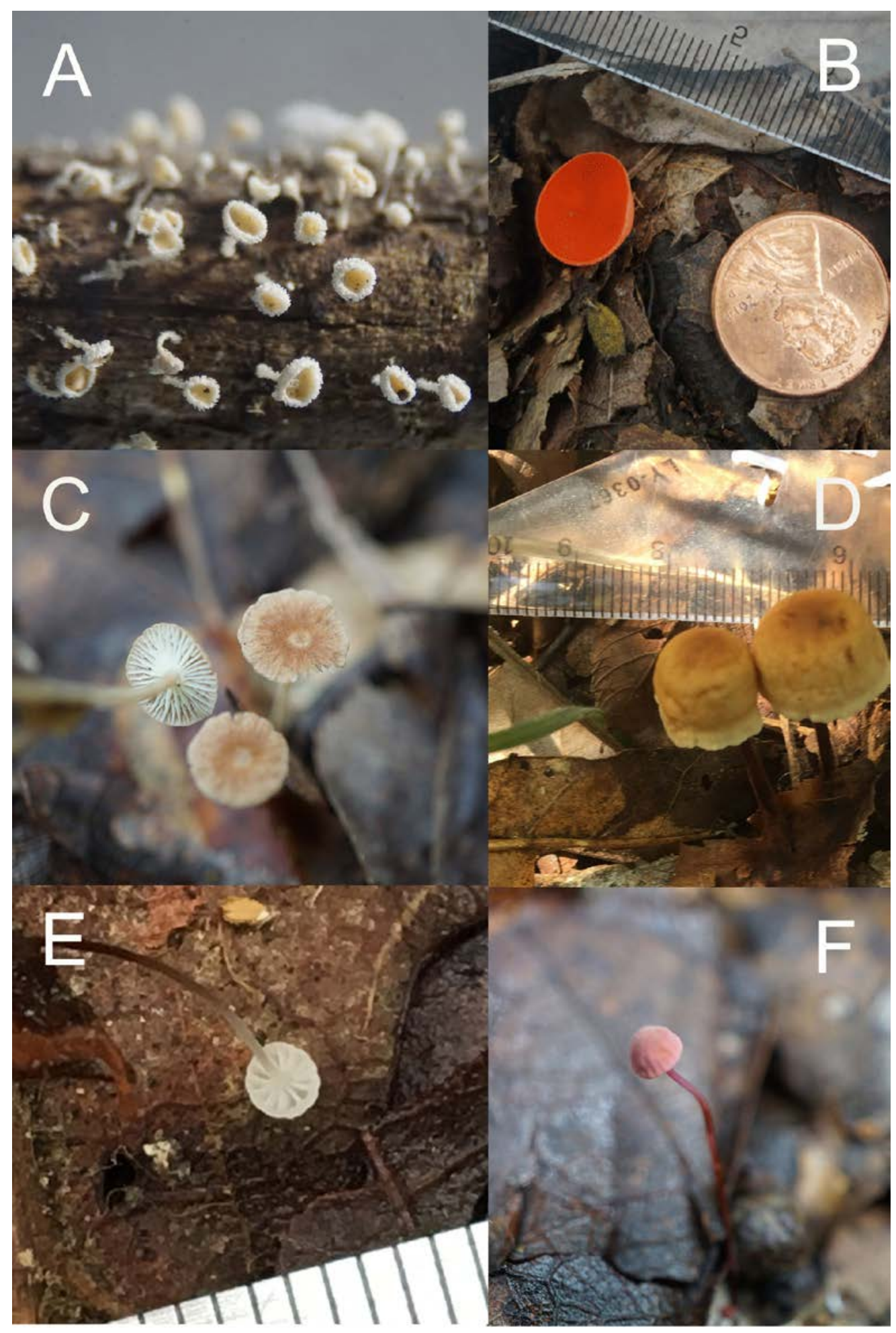

Fig. 3 - Selected species of litter-associated fungi recorded in the present study. A Lachnum virgineum. B Sarcoscypha occidentalis. C Gymnopus spongiocus. D Gymnopus disjunctus. E Mycena albiceps. F Marasmius pulcherripes.

As a general observation, saprophytic activity by fungi undoubtedly extends throughout the litter layer, but some degree of vertical stratification also exists, with the mycelia of ectomycorrhizal fungi likely to be confined to the lower humus/soil layer (O’Brien et al. 2005, Bahnmann et al. 
2018). One aspect of the ecology of the fungi associated with forest floor litter could not be addressed during the present study. This related to the temporal variation that occurs over the course of the fruiting season, as noted by Rudolph et al. (2018). Voříšková et al. (2014), who used a pyrosequencing technique, reported maxima in saprophytic genera on oak litter in the autumn, with ectomycorrhizal taxa more abundant in the summer. Just what seasonal differences exist in northwest Arkansas remains unknown. An appreciable number of fungi identified in the present study (a total of 342 specimens) could not be assigned to a functional group. It seems likely that many of these "incertaine sedis" fungi decompose litter, but other functional roles are possible, as evidenced by the presence of some taxa known to be mycoparasites. The presence of lichenized fungi in forest floor litter (Fig. 1) is probably the result of fragments derived from the thallus of one of these organisms on some other substrate (e.g., the bark of trees, where they are often quite common).

In conclusion, the results presented herein indicate that the fungi associated with forest floor litter are a diverse and dynamic assemblage (Fig. 3) whose contributions to the overall ecology of forest ecosystems should not be ignored, since they contribute to the structural stability, nutrient availability, productivity and other critical aspects of ecosystem functioning. The present study documented this assemblage of fungi for the first time in northwest Arkansas, using current molecular methods to identify the various taxa present. Many of these undoubtedly represent new records for both northwest Arkansas and the Ozark region of the central United States.

\section{Acknowledgements}

Appreciation is extended to the Iraqi government for the scholarship opportunity and financial support they have provided. Thanks are extended to personnel of the Pea Ridge National Military Park and Devil's Den State Park for giving us permission to carry out the research described herein. This research was supported in part by funds from the Slime Mold Project at the University of Arkansas. The first author also acknowledges the Delzie Demaree Research Grant received from the Arkansas Native Plant Society as well as the input provided by Don Nelsen, Ankush Nautiyal and Mourad Ben Hassine Ben Ali relating to laboratory equipment and protocols. Don Nelson also provided helpful comments on an earlier draft of this manuscript.

\section{References}

Bahnmann B, Mašínová T, Halvorsen R, Davey ML et al. 2018 - Effects of oak, beech and spruce on the distribution and community structure of fungi in litter and soils across a temperate forest. Soil Biology and Biochemistry 30(119), 162-73.

Baldrian P. 2017 - Forest microbiome: diversity, complexity and dynamics. FEMS Microbiology reviews 41(2), 109-130.

Berg B, McClaugherty C. 2003 - Plant Litter: Decomposition, Humus Formation, Carbon Sequestration. Springer Berlin Heidelberg, Germany.

Binion DE, Stephenson SL, Roody WC, Burdsall HH et al. 2008 - Macrofungi Associated with Oaks of Eastern North America. West Virginia University Press, Morgantown, West Virginia.

Boddy L, Frankland J, Van West P. 2008 - Ecology of Saprotrophic Basidiomycetes. British Mycological Society Symposia Series, Volume 28. Elsevier Academic Press, London.

Bödeker IT, Lindahl BD, Olson Å, Clemmensen KE. 2016 - Mycorrhizal and saprotrophic fungal guilds compete for the same organic substrates but affect decomposition differently. Functional Ecology 30(12), 1967-1978.

Breitenbach J, Kränzlin F. 1984 - Fungi of Switzerland, Volume 1, Ascomycetes. Verlag Mykologia, Switzerland.

Breitenbach J, Kränzlin F. 1995 - Fungi of Switzerland: A Contribution to the Knowledge of the Fungal Flora of Switzerland, Volume 4, Agarics 2. Verlag Mykologia, Switzerland. 
Cannon P, Sutton B. 2004 - Microfungi on wood and plant debris. In: Mueller G, Bills B, Foster M. (eds.) Biodiversity of Fungi: Inventory and Monitoring Methods. Elsevier Academic Press, Burlington Massachusetts.

Cebrian J. 1999 - Patterns in the fate of production in plant communities. The American Naturalist 154(4), 449-468.

Claridge AW, Trappe JM, Hansen K. 2009 - Do fungi have a role as soil stabilizers and remediators after forest fire? Forest Ecology and Management 257(3), 1063-1069.

Elliott TF, Stephenson SL. 2018 - Mushrooms of the Southeastern United States. Timber Press, Portland, Oregon.

Gardes M, Bruns TD. 1993 - ITS primers with enhanced specificity for basidiomycetes application to the identification of mycorrhizae and rusts. Molecular Ecology 2(2), 113-118.

Goswami M, Bhattacharyya P, Mukherjee I, Tribedi P. 2017 - Functional diversity: an important measure of ecosystem functioning. Advances in Microbiology 7(01), 82.

Hättenschwiler S, Tiunov AV, Scheu S. 2005 - Biodiversity and litter decomposition in terrestrial ecosystems. Annual Review of Ecology, Evolution, and Systematic 36, 191-218.

Hardoim PR, van Overbeek LS, Berg G, Pirttilä AM. 2015 - The hidden world within plants: ecological and evolutionary considerations for defining functioning of microbial endophytes. Microbiology and Molecular Biology Reviews 79, 293-320.

Jacobsen RM, Sverdrup-Thygeson A, Kauserud H, Birkemoe T. 2018 - Revealing hidden insectfungus interactions; moderately specialized, modular and anti-nested detritivore networks. Proceedings of the Royal Society B 285(1876), 20172833.

Kubartová A, Ranger J, Berthelin J, Beguiristain T. 2009 - Diversity and decomposing ability of saprophytic fungi from temperate forest litter. Microbial Ecology 58(1), 98-107.

Lodge DJ, Ammirati JF, O’Dell TE, Mueller GM et al. 2004 - Terrestrial and lignicolous macrofungi. In: Mueller G, Bills B, Foster M. (eds.) Biodiversity of Fungi: Inventory and Monitoring Methods. Elsevier Academic Press, Burlington Massachusetts.

O’Brien HE, Parrent JL, Jackson JA, Moncalvo JM, Vilgalys R. 2005 - Fungal community analysis by large-scale sequencing of environmental samples. Applied and Environmental Microbiology 71(9), 5544-5550.

Prakash CP, Thirumalai E, Rajulu MG, Thirunavukkarasu N, Suryanarayanan TS. 2015 - Ecology and diversity of leaf litter fungi during early-stage decomposition in a seasonally dry tropical forest. Fungal Ecology 17, 103-113.

Purahong W, Krüger D, Buscot F, Wubet T. 2016 - Correlations between the composition of modular fungal communities and litter decomposition-associated ecosystem functions. Fungal Ecology 22, 106-114.

Rinaldi AC, Comandini O, Kuyper TW. 2008 - Ectomycorrhizal fungal diversity: separating the wheat from the chaff. Fungal Diversity 33, 1-45.

Rudolph S, Maciá-Vicente JG, Lotz-Winter H, Schleuning M, Piepenbring M. 2018 - Temporal variation of fungal diversity in a mosaic landscape in Germany. Studies in Mycology 89, 95104.

Stephenson SL. 2010 - The Kingdom Fungi: The Biology of Mushrooms, Molds, and Lichens. Timber Press. Portland, Oregon.

Stephenson SL, Adams HS, Huebner CD. 2007 - Upland forest vegetation of the Ozark Mountains in northwestern Arkansas. Rhodora 109, 197-221.

Tedersoo L, Bahram M, Põlme S, Kõljalg U et al. 2014 - Global diversity and geography of soil fungi. Science 346 (6213), 1256688.

Voř́šsová J, Brabcová V, Cajthaml T, Baldrian P. 2014 - Seasonal dynamics of fungal communities in a temperate oak forest soil. New Phytologist 201(1), 269-278.

White TJ, Bruns TD, Lee SB, Taylor JW. 1990 - Amplification and direct sequencing of fungal ribosomal RNA genes for phylogenetics. In: Innis MA, Gelfand DH, Sninsky JJ, White TJ. (eds.). PCR Protocols: a Guide to Methods and Applications. Academic Press, San Diego, California. 